\title{
NEUROPSYCHOLOGICAL ASSESSMENT OF COGNITIVE DISORDERS IN LUPUS PATIENTS AT A TERTIARY HOSPITAL IN MANAUS/AM
}

\author{
Aline Oliveira Alves ${ }^{1, \star}$, Bárbara Seabra Carneiro², Helena Lúcia Alves Pereira² \\ 1.Universidade Nilton Lins, Manaus (AM), Brazil. 2.Universidade Federal do Amazonas, Manaus (AM), Brazil. \\ *Corresponding author: ally_oliveira21@hotmail.com
}

\section{BACKGROUND}

Systemic lupus erythematosus (SLE) is an inflammatory autoimmune disease that affects several organs and systems, including the central nervous system, which can cause cognitive damage to the patient. The present study is an analysis of the existence of cognitive disorders associated with SLE, through the application of neuropsychological tests and analysis of medical records, with the aim of determining the frequency and profile of cognitive impairment and verifying the relationship between cognitive impairment and disease activity according to SLEDAI.

\section{METHODS}

Cross-sectional study in which information collected from neuropsychological tests and review of medical records of a total of 100 patients diagnosed with SLE were analyzed, according to the classification criteria of the ACR 1997, aged between 18 and 60 years, treated at the rheumatology service in referral hospital. The instruments used to collect these data were: Mini-Mental State Examination (MMSE) and the clock-drawing test (CDT).

\section{RESULTS}

We identified 28 patients ( 3 men and 25 women), with a mean age of 38.2 years, who showed reduced performance in tests with scores below the cutoff values for both the MMSE and the CDT and the most prevalent form found was the mild cognitive impairment. In the MMSE, it was observed that the domain with the greatest changes was attention and calculation, which corresponds to a reduction in immediate and working memory, but there was also a reduction in short-term memory with the difficulty of recalling recent records, and also in the temporal-spatial orientation, while in the CDT it was demonstrated a reduction in the perceptualvisual, constructive and conceptual-abstract abilities. Of this total, $71.5 \%$ had 12 years of schooling or more, the average length of time with the disease was 9.5 years, $60.7 \%$ had SLEDAI scores compatible with active disease (mild - 35\%, moderate - $23,5 \%$, severe $-41 \%), 75 \%$ had other comorbidities, the most prevalent being systemic arterial hypertension.

\section{CONCLUSION}

The presence of cognitive disorders was evidenced in patients with SLE. Changes in neuropsychological tests added to other clinical information collected suggest that there may be a relationship between these disorders and activity, disease duration and the presence of other comorbidities.

\section{KEYWORDS}

Systemic lupus erythematosus, Cognitive disorders, Neuropsychiatric manifestations. 